\title{
High level evidence does not support first wave behavioural approaches to parent-infant sleep
}

\section{Dear Editor,}

I welcome Kempler et al.'s systematic review and meta-analysis of randomised controlled trials (RCTs) investigating whether psychosocial sleep interventions improve infant sleep or maternal mood in the postnatal period [1]. I have two points to make in response.

1) Kempler et al. state that their findings do not concur with the findings of Douglas and Hill's broader meta-narrative systematic review of behavioural sleep interventions in the first six months of life, because Kempler et al. found 'a clear effect of interventions on sleep administered in the perinatal period for infant nocturnal total sleep time.'

The authors are mistaken. Our systematic review also concludes (p. 499): 'Application of behavioural methods from the first weeks of life increases self-regulated sleep periods and increases total 24-h duration of time spent in the cot without signalling by 29 min' [2].

We then go on to argue that decreased episodes of nightwaking or longer infant sleep durations do not inevitably improve outcomes for mothers and their infants, as is often assumed.'

Kempler et al. similarly conclude: 'Psychosocial sleep interventions appear to impact the amount of sleep that a mother reports her baby to have, although the infants continue to wake as frequently. More research is needed to confirm whether sleeprelated improvements can translate into improvements in maternal mood.'

We chose to use a meta-narrative systematic review in order to make sense of the heterogenous literature concerning interventions for parent-infant sleep, given the limitations of RCTs for the investigation of complex clinical problems [3], and are interested to see that this quantitative meta-analysis corroborates our key findings.

2) Kempler et al. state that the psychosocial interventions under investigation are comprehensive multi-component interventions, and that it is not possible to distinguish between the effective components of the treatment. Unfortunately the authors fail to consider the unifying theoretical frame out of which these components arise.

DOI of original article: http://dx.doi.org/10.1016/j.smrv.2015.08.002.
All the RCTs analysed by Kempler et al. deliver strategies which arise from first wave behaviourism (FWB), a foundational psychology prominent in the 1950s and 1960s (FWB has subsequently evolved into second- and third-wave behaviourisms in other fields, but remains a dominant approach to the care of new families). FWB uses operant conditioning (unmodified or graduated extinction methods) to entrain an infant's neurobiological characteristics, in the belief that nocturnal signalling will occur less often, which will improve maternal mental health. 'Camping out' and 'controlled crying' are just two popular FWB programs. More broadly, FWB strategies include regulation of feed times, algorithms for sleep durations and bedtimes, lists of 'tired' cues, avoidance of 'overstimulation', strategies that aim to condition the infant to fall asleep in the absence of feeding or bodily contact with the carer, and delayed responses to cues or non-response to cues (e.g., ignoring the cue of sleepiness at the end of feeds and implementing feed-play-sleep cycles) [2,4-6].

In summary, Kempler et al.'s study confirms that high level evidence does not support the application of FWB approaches to parent-infant sleep, particularly in the first six months of life. Douglas and Hill's findings suggest that FWB approaches may be detrimental to some families.

Yet application of these interventions is widespread: even senior figures claim that not using them may be unethical, placing health professionals and parents under pressure to comply [6]. Clearly a paradigm shift is required in the management of parent-infant sleep problems. In our work, we have integrated third wave behaviourism with sleep science, neuroscience, and attachment literature to create a new paradigm for the support of families with infant sleep problems ('The Possums Sleep Intervention') [4]. This program is currently available online for parents at http://www. possumsonline.com/sleep-film, and is undergoing evaluation.

\section{References}

[1] Kempler L, Sharpe L, Miller CB, Bartlett DJ. Do psychosocial sleep interventions improve infant sleep or maternal mood in the postnatal period? A systematic review and meta-analysis of randomised controlled trials. Sleep Med Rev 2015;29:15-22.

[2] Douglas P, Hill PS. Behavioural sleep interventions in the first six months of life do not improve outcomes for mothers or infants: a systematic review. J Dev Behav Pediatr 2013:34:497-507.

[3] Greenhalgh T. Evidence-based medicine: a movement in crisis? BMJ 2014:348.

[4] Whittingham K, Douglas PS. Optimising parent-infant sleep from birth to 6 months: a new paradigm. Infant Ment Health J 2014;35:614-23.

[5] Douglas PS, Whittingham K. Response to 'Sleeping like a baby? Infant sleep impact on care givers and current controversies'. J Paediatr Child Health 2015;51:234. 
[6] Hiscock H, Fisher J. Sleeping like a baby? Infant sleep: impact on caregivers and current controversies. J Paediatr Child Health 2014;51:361-4.

Pamela S. Douglas

The Possums Clinic, Brisbane, Australia

Centre for Health Practice Innovation, Griffith University, Australia
Discipline of General Practice, The University of Queensland, Australia E-mail address: pameladouglas@uq.edu.au.

11 October 2015 Available online $\mathrm{xxx}$

Please cite this article in press as: Douglas PS High level evidence does not support first wave behavioural approaches to parent-infant sleep, Sleep Medicine Reviews (2015), http://dx.doi.org/10.1016/j.smrv.2015.10.007 